\title{
Simulação da rentabilidade e viabilidade econômica de um modelo de produção de leite em free-stall
}

\author{
[Simulation of economic feasibility and profitability of a free stall model milk production] \\ I.D. Barbosa Silveira ${ }^{1}$, M.D.P. Peters ${ }^{2 *}$, T. Storch ${ }^{3}$, E.A. Ziguer ${ }^{4}$, V. Fischer ${ }^{5}$ \\ ${ }^{1}$ Departamento de Zootecnia, Universidade Federal de Pelotas - Pelotas, RS \\ ${ }^{2}$ Pós-Graduação em Zootecnia - Universidade Federal de Pelotas - Pelotas, RS - Bolsista CAPES \\ ${ }^{3}$ Médico veterinário \\ ${ }^{4}$ Aluno de graduação - Universidade Federal de Pelotas- Pelotas, RS \\ ${ }^{5}$ Departamento de Zootecnia - Universidade Federal do Rio Grande do Sul
}

\begin{abstract}
RESUMO
Avaliaram-se a rentabilidade e a viabilidade econômica da implantação de um sistema de produção de leite em free-stall. Foi realizada uma pesquisa técnico-mercadológica para definir os investimentos necessários e determinar os custos de cada fator de produção. Considerou-se um modelo de produção composto por 100 vacas em lactação, com produção média total de 2595 litros de leite por dia. O custo operacional total mensal foi de $\mathrm{R} \$ 27.787,39$, tendo a nutrição como a variável de maior relevância, representando 62,7\% do custo. Para a receita bruta mensal, obteve-se o valor de $\mathrm{R} \$ 43.817,50$ e a consequente receita líquida mensal de $\mathrm{R} \$ 16.030,11$. Observou-se rentabilidade de $12,2 \%$ ao ano, com valor presente líquido negativo de $\mathrm{R} \$ 131.627,39$. Concluiu-se que o modelo proposto mostrou-se economicamente inviável levando-se em consideração os preços estimados.
\end{abstract}

Palavras-chave: produção de leite, free-stall, rentabilidade, simulação

\begin{abstract}
The economical profitability and viability of the implantation of a milk production system in free stall were evaluated. A technical-market analysis was done to define the necessary investment as well as to determine the costs of each production factor. A production model with 100 lactating cows averaging 2,595 liters of daily production was considered. The total monthly operational cost was R $\$ 27,781.39$ showing that nutrition is the variable with higher relevance (62.7\% of the cost). For the monthly gross income a value of $R \$ 43,817.50$ was obtained and, consequently, the monthly net income (NI)was $R \$ 16,030.11$. A 12.2\% profitability per year from NI rendering a deficit net value of $R \$ 131,627.39$. Considering the estimated prices it is concluded that the proposed model was economically unfeasible.
\end{abstract}

Keywords: milk yield, free stall, profitability, simulation

\section{INTRODUÇÃO}

Uma nova realidade na atividade leiteira tem surgido com a conscientização dos produtores da necessidade de administrarem eficientemente a atividade, tornando-a mais competitiva e buscando maior rentabilidade. A eficiência no processo produtivo é um ponto fundamental para a manutenção de qualquer atividade pecuária, sendo que estudos com a finalidade de determinar o custo e a rentabilidade proporcionam aos produtores subsídios para a tomada de decisões (Ferreira et al., 2004).

Dados obtidos da apuração dos custos de produção têm sido utilizados para análise da rentabilidade, redução dos custos controláveis, planejamento e controle das operações do

Recebido em 10 de março de 2010

Aceito em 28 de março de 2011

*Autor para correspondência (corresponding author)

E-mail: monipaulapeters@yahoo.com.br 
sistema e identificação do ponto de equilíbrio da atividade (Lopes e Carvalho, 2000). Porém, pesquisadores têm se preocupado em estimar o custo da produção e estudar a viabilidade econômica da produção de leite (Marques, 1999), sem, contudo, avaliar o impacto da variação do preço do leite e dos fatores de produção na rentabilidade do sistema.

Segundo Lopes e Carvalho (2000), a análise econômica da atividade leiteira é importante, pois é possível detectar os pontos críticos do sistema produtivo, buscando soluções para eles, a fim de aumentar os lucros ou diminuir os custos de produção em sistemas intensivos. Este trabalho teve como objetivo avaliar a rentabilidade e a viabilidade econômica da implantação de um sistema simulado de produção de leite em free-stall, avaliando-se a sensibilidade dos indicadores econômicos.

\section{MATERIAL E MÉTODOS}

O estudo teve como base a simulação da implantação de um sistema de produção de leite em free-stall para 100 animais da raça Holandesa. Realizou-se uma pesquisa técnicomercadológica com o objetivo de definir os investimentos necessários para a implantação do sistema, bem como para determinar os custos de cada fator de produção e a possibilidade de negociação da matéria-prima com laticínios regionais capazes de absorver o volume produzido. Os investimentos totalizaram R\$937.636,76 referentes à aquisição de 115 animais, instalações do free-stall, sala de ordenha, maquinários, entre outros, conforme descrito na Tab. 1. Dentre os investimentos, também foi considerado o capital de giro no valor de R\$27.787,39, necessário para manutenção da atividade durante o primeiro mês, visto que neste período a atividade não geraria receita.

Tabela 1. Investimentos necessários para a implantação de sistema simulado de produção de leite em freestall

\begin{tabular}{lccr}
\multicolumn{1}{c}{ Equipamentos } & Quantidade & Preço (R\$) & \multicolumn{1}{c}{$\begin{array}{c}\text { Investimento final } \\
(\mathrm{R} \$)\end{array}$} \\
\hline Arado reversível - 3 discos & 1 & $3.500,00$ & $3.500,00$ \\
Balança - 250kg & 1 & 300 & 300,00 \\
Capital de giro & - & - & $27.787,39$ \\
Computador & 1 & $2.500,00$ & $2.500,00$ \\
Concha carregadeira & 1 & $4.000,00$ & $4.000,00$ \\
Ensiladeira linha simples & 1 & $17.000,00$ & $17.000,00$ \\
Esterqueira & 1 & $15.000,00$ & $15.000,00$ \\
Estrutura de escritório e farmácia & 1 & $10.000,00$ & $10.000,00$ \\
Estrutura de free-stall & 1 & $150.000,00$ & $150.000,00$ \\
Gerador de energia & 1 & $2.500,00$ & $2.500,00$ \\
Grade de disco 26 lam & 1 & $4.000,00$ & $4.000,00$ \\
Máquina pré-secado & 1 & $41.000,00$ & $41.000,00$ \\
Misturador de ração & 1 & $5.000,00$ & $5.000,00$ \\
Misturador total mix & 1 & $30.000,00$ & $30.000,00$ \\
Outros & - & $44.649,37$ & $44.649,37$ \\
Plantadeira & 1 & $30.000,00$ & $30.000,00$ \\
Reboque & 2 & $3.000,00$ & $6.000,00$ \\
Resfriador expansão - 3000L & 1 & $18.000,00$ & $18.000,00$ \\
Sala de ordenha 2x6 & 1 & $28.000,00$ & $28.000,00$ \\
Silos trincheira & 11 & 400,00 & $4.400,00$ \\
Trator usado & 1 & $20.000,00$ & $20.000,00$ \\
Trator novo & 1 & $120.000,00$ & $120.000,00$ \\
Triturador & 1 & $4.000,00$ & $4.000,00$ \\
Tronco de manejo & 115 & $5.000,00$ & $5.000,00$ \\
Vacas em início de lactação & & $3.000,00$ & $345.000,00$ \\
\hline Investimento total & & $937.636,76$ \\
\hline
\end{tabular}


A estrutura do free-stall foi projetada a partir de valores médios indicados para o sistema em que a unidade seria composta por uma sala de ordenha do tipo espinha de peixe $2 \times 6$ canalizada, sala de máquinas e leite, escritório e farmácia, duas salas de espera pré-ordenha e quatro baias com capacidade de 25 animais cada. A ocupação das baias seria determinada a partir da categoria e do estádio lactacional, com o objetivo de maximizar as eficiências produtiva e nutricional, assim consideradas: baia 1 - composta por animais de terceira, quarta e quinta ordem de lactação e com até 100 dias de lactação; baia 2 animais de terceira, quarta e quinta ordem de lactação e com 101 a 205 dias de lactação; baia 3 - animais de terceira, quarta e quinta ordem de lactação e com 206 a 315 dias de lactação; baia 4 - composta por primíparas (primeira lactação) e vacas de segunda ordem de lactação, com estádios de lactação variados; baia 5 - composta por vacas secas.
A dieta foi calculada com base no NRC (Nutrient..., 2001), utilizando-se, a produção leiteira estimada de acordo com o período lactacional e o peso médio de 550 e $650 \mathrm{~kg}$ para vacas primíparas e multíparas, respectivamente. Foi estimada a produtividade média de 34, 27, 18 e 20 litros/animal/dia para as baias 1, 2, 3 e 4, respectivamente. A ingestão de matéria seca (IMS) foi de 3,2\%, 3,3\%, 2,8\% e 3,0\%, seguindo-se a mesma ordem anterior. Na baia 5 , composta pelas vacas secas, a IMS foi de $2 \%$, e os animais foram alimentados em uma área exterior, anexa ao free-stall. A composição nutricional específica para cada grupo encontrase na Tab. 2. O custo de cada ingrediente da dieta foi estimado com base em valores médios; para os principais componentes do concentrado, milho e soja, utilizou-se a média histórica dos últimos 10 anos, a fim de se minimizar o efeito da variação dos preços desses produtos (Anuário..., 2004).

Tabela 2. Composição (\%) e custo da dieta para vacas leiteiras, de acordo com cada baia

\begin{tabular}{lcccccc}
\multicolumn{1}{c}{ Ingrediente } & Custo/ & Baia & Baia & Baia & Baia & Baia \\
& $\mathrm{kg} / \mathrm{MS}$ & 1 & 2 & 3 & 4 & 5 \\
\hline Pré-secado & 0,08 & - & 21,31 & 31,79 & 33,41 & 77,75 \\
Silagem de milho & 0,14 & 45,03 & 42,01 & 37,17 & 31,53 & - \\
Grão de milho triturado & 0,31 & 27,68 & 17,70 & 14,84 & 18,68 & 10,31 \\
Grão de soja triturado & 0,59 & 18,61 & 17,86 & 14,78 & 15,10 & - \\
Farelo de soja & 0,67 & 6,39 & - & - & - & - \\
Casca de soja & 0,21 & - & - & - & - & 10,31 \\
Bicarbonato de sódio & 1,50 & 1,00 & - & - & - & - \\
Premix mineral e vitamínico & 1,40 & 1,29 & 1,13 & 1,42 & 128 & 1,62 \\
IMS animal/dia & & 20,87 & 21,19 & 18,35 & 18,21 & 12,80 \\
IMS (\%PV) & & 3,2 & 3,3 & 2,8 & 3,0 & 2,0 \\
Custo total animal/dia (R\$) & & 6,99 & 5,36 & 4,22 & 4,28 & 1,74 \\
\hline
\end{tabular}

IMS: ingestão de matéria seca; PV: peso vivo. 1: animais de terceira, quarta e quinta ordem de lactação e com até 100 dias de lactação; baia 2: animais de terceira, quarta e quinta ordem de lactação e com 101 a 205 dias de lactação; baia 3: animais de terceira, quarta e quinta ordem de lactação e com 206 a 315 dias de lactação; baia 4: primíparas (primeira lactação) e vacas de segunda ordem de lactação variados; baia 5: vacas secas.

Nos custos operacionais totais (COT), foram considerados os gastos com nutrição, salários de quatro funcionários, um gerente e três empregados, imposto territorial rural (ITR), água, combustível, material para higienização, luz, medicamentos e gastos reprodutivos, assistência técnica e possíveis gastos com manutenção de equipamentos. Para cálculo do salário dos funcionários, foi utilizado um acréscimo de 58\% referente aos encargos sociais com alíquotas de 20\%, $8 \%$ e $30 \%$, respectivamente, para INSS, FGTS e insalubridade (Zainaghi, 2003).
A reposição dos animais foi considerada como uma despesa anual, pois a venda dos animais de descarte participava na composição da receita bruta (RB) e, assim, abatia parte do valor a ser investido. Foi estimado o preço médio de compra de $\mathrm{R} \$ 2.500,00 / a n i m a l$, perfazendo, dessa forma, uma despesa adicional de $\mathrm{R} \$ 37.500,00$ ao ano. A $\mathrm{RB}$ foi determinada a partir do volume de leite mensal produzido somada à venda de animais de descarte, em que foi considerado o valor médio de $\mathrm{R} \$ 800,00 /$ animal. Considerou-se o preço de $\mathrm{R} \$ 0,55 /$ litro de leite como o valor médio para este nicho de produtor. 
Determinou-se a receita líquida (RL) subtraindose RB da COT, utilizada para cálculo dos seguintes indicadores econômicos: valor presente líquido (VPL) e taxa interna de retorno (TIR). Foi utilizado um horizonte de planejamento como base de cálculo de 15 anos, pois, neste período, a maior parte dos bens sofreu depreciação (Buarque, 1984). Dessa forma, ao final do estudo, foi incluída na RL uma alíquota estimada em 20\% dos investimentos iniciais, referente ao valor residual dos equipamentos que ainda não apresentavam depreciação completa, bem como o valor referente ao retorno do capital de giro.

O VPL indica se os retornos obtidos com o investimento serão suficientes para compensar os custos operacionais e o investimento realizado em valores presentes, ou seja, para que o investimento seja considerado satisfatório, seu resultado deve ser positivo. A rentabilidade do projeto foi determinada a partir da TIR, assim, para que o investimento seja satisfatório, a TIR deve ser superior à taxa mínima de atratividade (TMA), ou seja, maior que o rendimento de uma aplicação bancária de segurança e liquidez, como a caderneta de poupança ou um certificado de depósito bancário (Buarque, 1984). Para base de cálculo, foi utilizada a TMA de 15\% ao ano.

\section{RESULTADOS E DISCUSSÃO}

Foi obtido como COT mensal o valor de $\mathrm{R} \$ 27.787,39$, indicando-se a representatividade de cada variável na Tab.3. Para a RB mensal, obteve-se o valor de $\mathrm{R} \$ 43.817,50$ e consequente $\mathrm{RL}$ mensal de $\mathrm{R} \$ 16.030,11$. A partir desta RL, alcançou-se a rentabilidade de $12,2 \%$ ao ano, com VPL negativo de $\mathrm{R} \$ 131.627,39$. Em tal contexto, este estudo simulado mostrou-se inviável economicamente, ou seja, renderia $\mathrm{R} \$ 131.627,39$ a menos que uma aplicação financeira $(\mathrm{TMA}=15 \% / \mathrm{ano})$, no período de 15 anos. Fellet e Galan (2000) encontraram rentabilidade de 10,0\% ao ano em sistemas de produção que utilizavam como base nutricional cana-de-açúcar, silagem de milho e suplementação com concentrado. Dados semelhantes foram observados por Schiffler et al. (1999), os quais, em avaliação de sistemas semiintensivos, verificaram remuneração sobre o capital investido de 9,8\%. Portanto, torna-se evidente que o modelo proposto foi mais favorável que os sistemas de produção menos intensivos, pois, segundo Konzen (1998), os sistemas mais tecnificados de produção de leite são mais rentáveis que os menos tecnificados.

Tabela 3. Custo operacional total mensal e representação de cada variável em sistema simulado de produção de leite em free-stall

\begin{tabular}{lrr} 
Custo fixo & Mensal total, R\$ & $\%$ \\
\hline Salário dos funcionários & $4.266,00$ & 15,35 \\
Imposto Territorial Rural & 75,00 & 0,27 \\
Subtotal & $4.341,00$ & 15,62 \\
\hline Custo variável & & 1,12 \\
\hline Água & 310,50 & 1,80 \\
Assistência técnica & 500,00 & 2,33 \\
Combustível & 646,38 & 7,38 \\
Higienização & $2.050,00$ & 2,91 \\
Luz & 810,00 & 3,60 \\
Manutenção & $1.000,00$ & 2,52 \\
Medicamentos e reprodução & 701,08 & 62,72 \\
Nutrição & $17.428,44$ & 84,38 \\
Subtotal & $23.446,39$ & 100,00 \\
\hline COT & $27.787,39$ & \\
\hline
\end{tabular}

Dentre os componentes do COT, a nutrição foi a variável de maior influência, representando 62,7\%. Ao se considerarem apenas os custos variáveis totais (CVT), a alimentação foi responsável por $71,5 \%$ destes custos. Resultados semelhantes foram encontrados por Lopes et al. (2005), os quais relataram que a alimentação de vacas de leite totalmente confinadas representa $66,5 \%$ do custo operacional efetivo. Ao se avaliarem de forma isolada os custos 
nutricionais, observaram-se as seguintes proporções médias para cada ingrediente: grão de soja $34,5 \%$, grão de milho $23,0 \%$, silagem de milho $19,6 \%$, pré-secado 9,1\%, sal mineral 7,5\%, farelo de soja 3,8\%, bicarbonato de sódio 1,3\% e casca de soja $1,1 \%$.

Dada a alta contribuição da nutrição para o COT, e levando-se em consideração que o grão de soja, o milho e a silagem são as principais variáveis determinantes deste custo, pode-se inferir que distorções de preços destes insumos podem influenciar de maneira significativa os custos de produção e, consequentemente, a resposta econômica do sistema produtivo adotado. A fim de quantificar a influência da variação de preços destes insumos, foram elaborados diferentes cenários de preços pagos por estes produtos, com o intuito de determinar as possíveis alterações nos indicadores financeiros.

Com base no que é apresentado na Fig. 1, podese inferir que, com os preços considerados para os componentes nutricionais, chega-se à rentabilidade de 12,2\% ao ano; com diminuição de $15 \%$ nos custos médios dos componentes da dieta, a TIR passa a 15,1\% e o VPL torna-se positivo em, aproximadamente, R\$4.704,61, o que viabiliza o sistema adotado. No entanto, os preços médios utilizados para os grãos de milho e soja encontram-se valorizados em relação ao presente momento, dessa forma, os indicadores financeiros com valores de hoje demonstrar-seiam ainda mais expressivos no aspecto de viabilidade financeira.

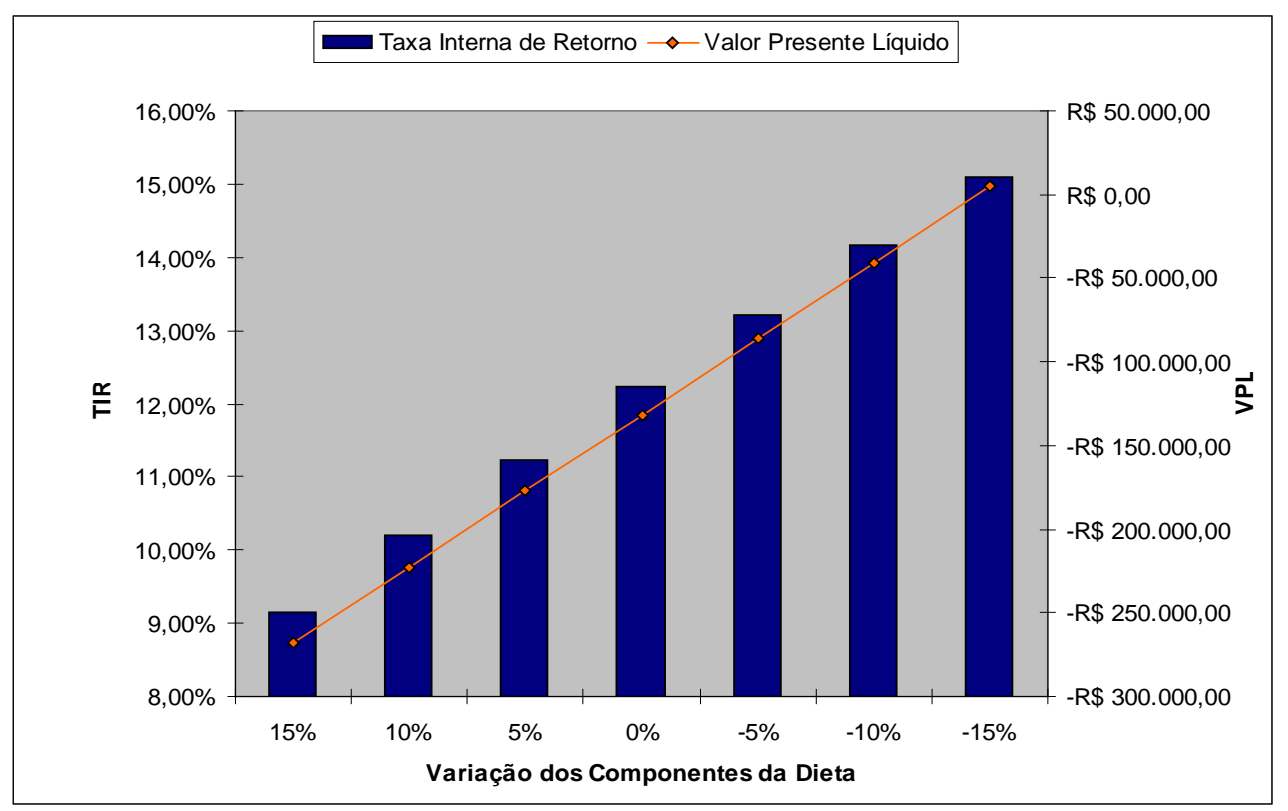

Figura 1. Simulação do impacto econômico - taxa interna de retorno (TIR) e valor presente líquido (VPL) - resultante da variação de preços dos componentes nutricionais em sistema simulado de produção de leite em free-stall.

Devido ao fato de o modelo apresentar como principal fonte de volumoso a silagem de milho, e esta ser susceptível a fatores bióticos e abióticos, como, por exemplo, à variabilidade climática de cada região, ocorre também grande variação no custo de sua produção (Faturi et al., 2003). Neste contexto, diferentes custos de produção para este produto foram simulados, conforme descrito na Fig. 2.
Torna-se evidente que alterações no custo de produção da silagem influenciam de forma significativa os indicadores econômicos. Adotando-se estratégias que visem à redução de custos, seja pelo gerenciamento da produção, seja pelo aumento da produtividade, podem-se alcançar respostas econômicas diferentes em, aproximadamente, R\$250.000,00. Por exemplo, 
custo aproximado de $\mathrm{R} \$ 0,03$ por kg de matéria verde (MV) poderia ser alcançado mediante uso de adubos orgânicos oriundos de dejetos dos animais (Lopes e Magalhães, 2005). Mais ainda, podem-se obter preços mais elevados em ocasiões decorrentes de transtornos climáticos. No entanto, despesas que gerem a necessidade de irrigação e também gastos inesperados de controle de pragas podem atuar em sentido contrário.

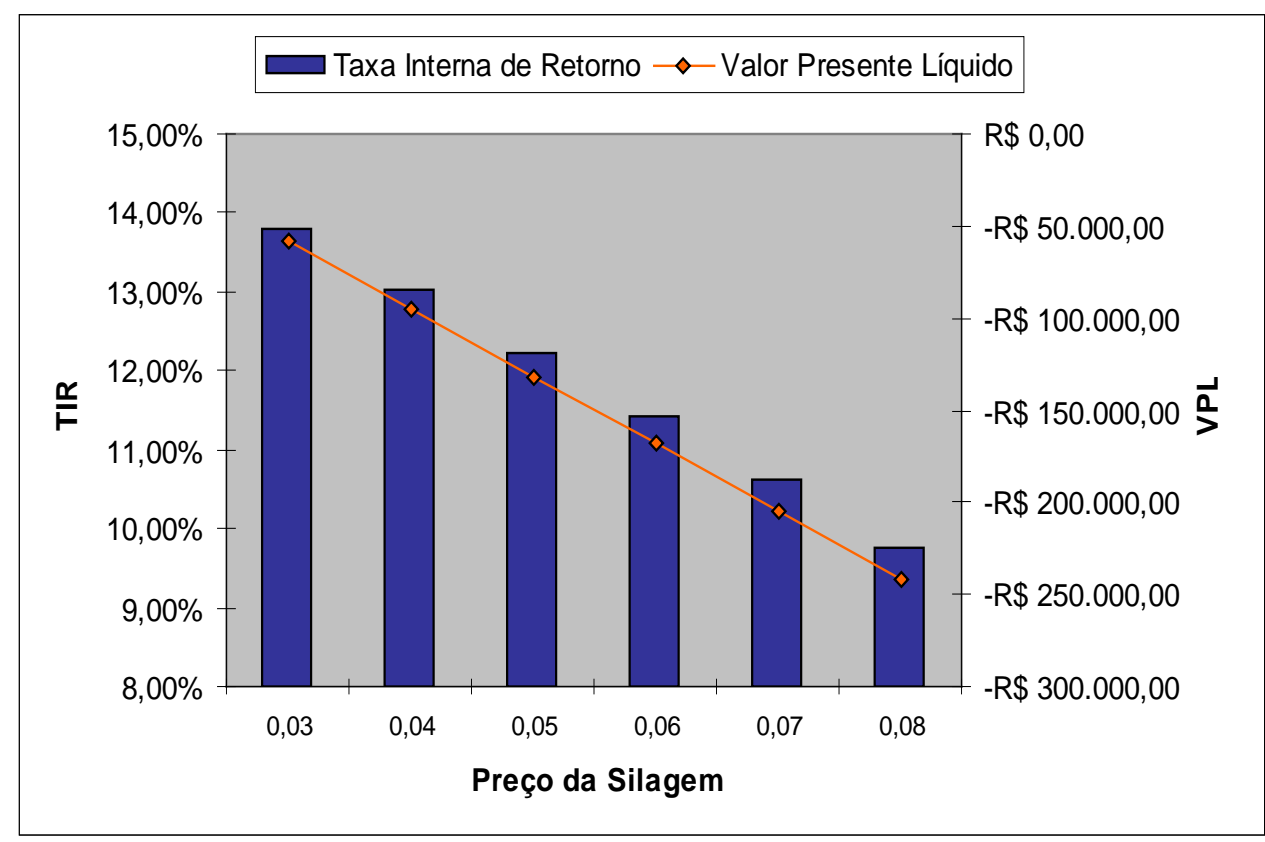

Figura 2. Simulação do custo da silagem de milho (R\$) na taxa interna de retorno (TIR) e impacto no valor presente líquido (VPL) em sistema simulado de produção de leite em free-stall.

Outra variável de relevância foi os gastos com funcionários, que representaram 98,3\% e 15,4\% dos custos fixos (CF) e COT, respectivamente. Nesse contexto, Lopes et al. (2001) verificaram que a mão de obra contribuiu com $25,8 \%$ do custo operacional efetivo, com relação vaca:homem de 7:1. Assim, neste estudo, a adoção do sistema intensivo de produção com relação vaca: homem de 28:1 possibilitou a diluição desses custos devido à intensificação da atividade. Lopes et al. (2005), ao avaliarem a representatividade da mão de obra em sistemas de alta adoção tecnológica, observaram que essa variável foi responsável por $14,1 \%$ do custo total, ou seja, valor semelhante aos encontrados no presente modelo de produção.

Com relação à origem das receitas, a RB gerada a partir da venda do leite representaria 97,7\% e, assim, os outros $2,3 \%$ da receita seriam originários da venda de animais de descarte. Portanto, qualquer alteração no preço de negociação na venda do produto leite pode influenciar e distorcer, de forma expressiva, os resultados econômicos do sistema

A fim de avaliar essas alterações, simulou-se a variação dos indicadores financeiros para diferentes cenários, referentes ao preço pago pelo litro do leite.

Conforme mostra a Fig. 3, observou-se grande sensibilidade na alteração dos indicadores financeiros, pois, com o acréscimo de $\mathrm{R} \$ 0,04$ na comercialização do produto, a rentabilidade passou a 15,7\%, obtendo-se o ganho de R\$33.593,43 sobre a aplicação financeira com TMA de $15 \%$ ao ano. Os principais motivos que explicam essa grande alteração são o grande volume produzido, bem como o fato de a venda do leite ser a principal fonte geradora de receita, conforme já comentado anteriormente. Dessa forma, fica evidente a dependência da negociação do produto com as indústrias, frente ao sucesso da atividade leiteira. 


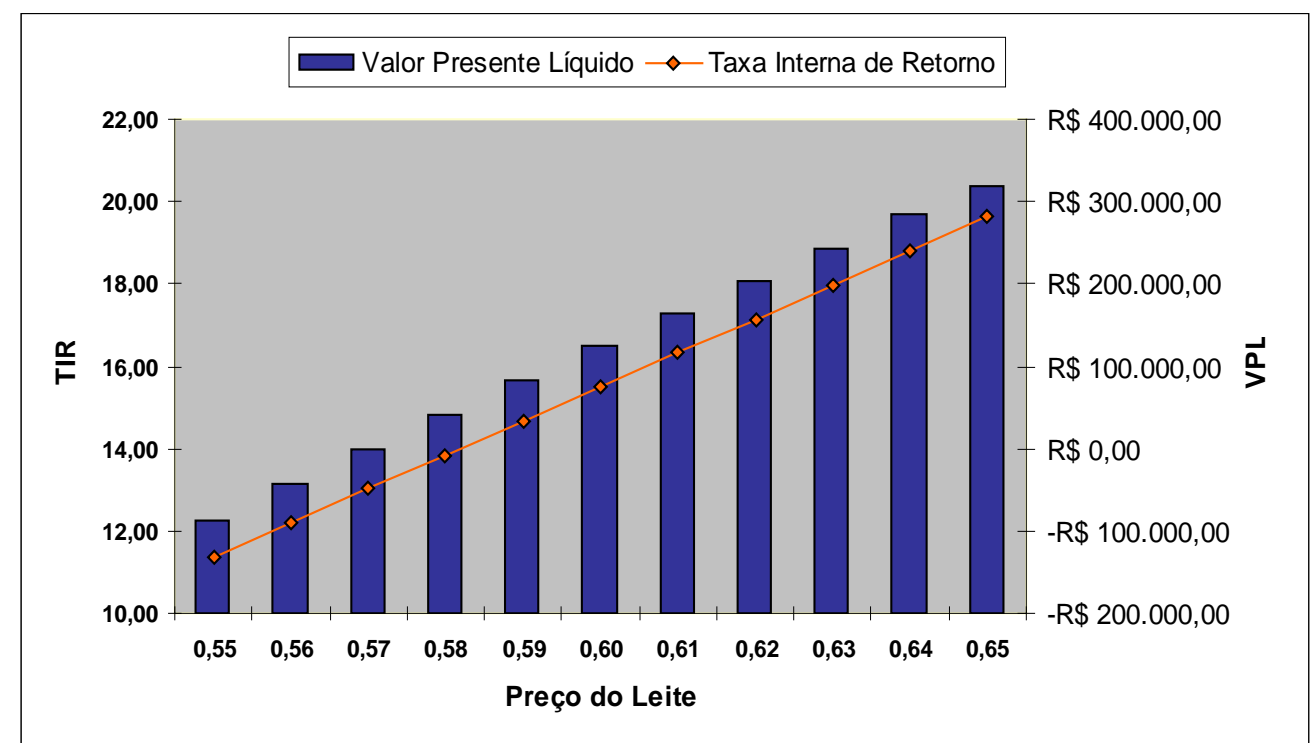

Figura 3. Alterações dos indicadores financeiros - taxa interna de retorno (TIR) e valor presente líquido (VPL) - vinculados às alterações dos preços do litro do leite (R\$) em sistema simulado de produção de leite em free-stall.

\section{CONCLUSÃO}

A implantação do modelo de sistema de produção de leite em free-stall demonstrou ser inviável com os preços considerados neste estudo.

\section{REFERÊNCIAS BIBLIOGRÁFICAS}

ANUÁRIO DA AGRICULTURA BRASILEIRA. São Paulo: FNP Consultoria e Comércio, 2004. 496p.

BUARQUE, C. Avaliação econômica de projetos. Rio de Janeiro: Campus, 1984. 266p.

FATURI, C.; RESTLE, J.; PASCOAL, L.L. et al Avaliação econômica de dietas com diferentes níveis de substituição do grão de sorgo por grão de aveia preta para terminação de novilhos em confinamento. Cienc. Rural, v.33, p.937-942, 2003.

FELLET, V.K.; GALAN, V.B. Diagnóstico e acompanhamento financeiro da atividade

leiteira. Rev. Preços Agric., v.14, p.14-17, 2000.

FERREIRA, M.M.; FERREIRA, A.C.M.; EZEQUIEL, J.M.B. Avaliação econômica da produção de bovinos confinados: estudo de caso. Inf. Econ., v.1, p.7-20, 2004.

KONZEN, O.G. Modernização e competitividade entre sistemas na produção de leite. Rev. Econ. Sociol. Rural, v.36, p.105-125, 1998.

LOPES, M.A.; CARVALHO, F.M. Custo de produção do leite. Bol. Agropecu., n.32, 2000. 42 p.
LOPES, M.A.; ALMEIDA JUNIOR, G.A.; CARVALHO, F.C. et al. Estudo da rentabilidade de um sistema de produção de leite tipo B no estado de São Paulo. In: CONGRESSO BRASILEIRO DE ADMINISTRAÇÃO RURAL, 4., 2001, Goiânia. Anais... Goiânia: ABAR, 2001. CD-ROM.

LOPES, M.A.; LIMA, A.L.R.; CARVALHO, F.M. et al. Resultados econômicos de sistemas de produção de leite com diferentes níveis tecnológicos na região de Lavras, MG. Arq. Bras. Med. Vet. Zootec., v.57, p.485-493, 2005.

LOPES, M. A.; MAGAHÃES, G. P. Análise da rentabilidade de terminação de bovinos de corte em condições de confinamento: um estudo de caso. Arq. Bras. Med. Vet. Zootec., v.57, 374-379, 2005.

MARQUES, V.M. Custos e escala na pecuária leiteira: estudo de casos. 1999. 59f. Dissertação (Mestrado em Administração Rural) - Universidade Federal de Lavras, Lavras, MG.

NUTRIENT requirements of dairy cattle. 7.ed. Washington, DC: National Academy of Sciences, 2001. 381p.

SCHIFFLER, E.A.; MÂNCIO, A.B.; GOMES, S.T. et al. Efeito da escala de produção nos resultados econômicos da produção de leite B no Estado de São Paulo1. Rev. Bras. Zootec., v.28, p.425-431, 1999.

ZAINAGHI, D.S. Curso de legislação social. 10.ed. São Paulo: Atlas, 2003. 